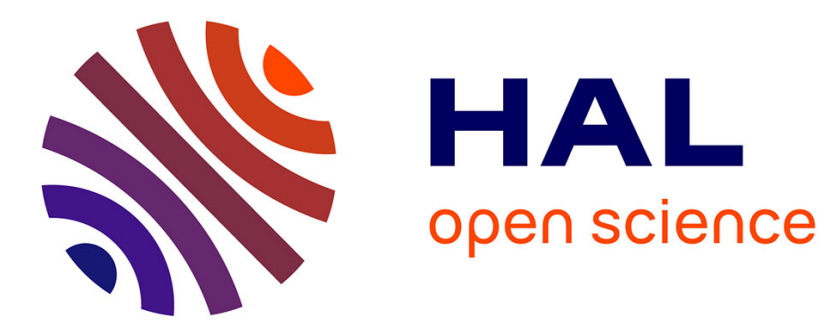

\title{
Nondipole effects in helium photoionization
}

Luca Argenti, Roberto Moccia

\section{To cite this version:}

Luca Argenti, Roberto Moccia. Nondipole effects in helium photoionization. Journal of Physics B: Atomic, Molecular and Optical Physics, 2010, 43 (23), pp.235006. 10.1088/0953-4075/43/23/235006 . hal-00576256

\section{HAL Id: hal-00576256 https://hal.science/hal-00576256}

Submitted on 14 Mar 2011

HAL is a multi-disciplinary open access archive for the deposit and dissemination of scientific research documents, whether they are published or not. The documents may come from teaching and research institutions in France or abroad, or from public or private research centers.
L'archive ouverte pluridisciplinaire HAL, est destinée au dépôt et à la diffusion de documents scientifiques de niveau recherche, publiés ou non, émanant des établissements d'enseignement et de recherche français ou étrangers, des laboratoires publics ou privés. 


\title{
Nondipole effects in helium photoionization
}

\author{
Luca Argenti \\ Atomic Physics, Fysikum, Stockholm University, AlbaNova University Center, \\ SE-106 91 Stockholm, Sweden \\ E-mail: argenti@physto.se \\ Roberto Moccia \\ IPCF, CNR, Via G. Moruzzi 1, 56100 Pisa, Italy \\ E-mail: moccia@ipcf.cnr.it
}

\begin{abstract}
An accurate calculation of the nondipole anisotropy parameter $\gamma$ in the photoionization of helium below the $\mathrm{N}=2$ threshold is presented. The calculated results are in fairly good agreement with the experimental results of Krässig et al [Phys. Rev. Lett. 88 (2002) 203002], but not as good as the accuracy of the calculation should have warranted. A careful examination of the possible causes for the observed discrepancies between theory and experiment seems to rule out any role either of the multipolar terms higher than the electric quadrupole, or of the singlet-triplet spin-orbit mixing. It is argued that such discrepancies might be instead of instrumental origin, due to the difficulty of measuring vanishingly small total cross sections $\sigma_{\text {tot }}$ with the required accuracy. In such eventuality, it might be more convenient to use a parameter other than $\gamma$, as for instance the drag current, to measure the nondipole anisotropy of the photoelectron angular distribution.
\end{abstract}

PACS numbers: $32.80 . \mathrm{Fb}$

Submitted to: J. Phys. B: At. Mol. Opt. Phys.

\section{Introduction}

For photoionization processes in the sub-keV X-ray energy range, the wavelength of the impinging radiation is significantly larger than the characteristic size of an atomic target in its ground state. This generally justifies the use of the time honored dipole approximation. In 1969, though, a net forward drift of the electrons ejected in xenon photoionization with lowenergy X-rays was detected [1]. For linearly polarized radiation, the dipole approximation (DA) predicts a symmetric angular distribution of the photoelectrons about the polarization vector. Beyond the DA, this symmetry no longer holds, possibly resulting in a net excess of photoelectrons along the direction of propagation of the impinging light. Therefore, the measurement reported in [1] was readily recognized as the first clear evidence of a deviation from the DA in atomic photoionization at low energies [2]. This and other similar results [3] were the basis for a systematic investigation of nondipole effects at energies so low that the wavelength of the radiation is orders of magnitude larger than the characteristic size of the target atom. The limits of the DA are emphasized whenever the dipole transition amplitude is vanishingly small, as it may occur near the zeroes of Fano profiles, or near Cooper minima, 
a common feature in the spectra of the heavier rare gases. Large nondipolar transition amplitudes, found for example near quadrupolar resonances, also cause the breakdown of the DA. Indeed, resonant nondipole effects at low photon energies can be comparable to nonresonant ones in the keV energy region $[4,5]$. Nondipole effects were eventually reported also in the photoionization of helium [6], the lightest of all rare gases and therefore arguably the least prone to give rise to observable nondipole effects. In that work, the authors were able to reveal the detailed resonant profile of the backward/forward asymmetry parameter $\gamma[7]$ in the vicinity of a dipole forbidden quadrupole-allowed resonance lying close to one which is dipole allowed.

In this paper we present the results of an accurate calculation of the asymmetry of the photoelectron angular distribution in the photoionization of helium in a large energy range below the $\mathrm{N}=2$ threshold comprising several closely spaced ${ }^{1} \mathrm{P}^{o}$ and ${ }^{1} \mathrm{D}^{e}$ doubly excited states. The results for the lowest couple of these resonances compare fairly well to the available experimental data [6]. Discrepancies between theory and experiment, though, are found in correspondence to the minimum of the total cross section $\sigma_{\text {tot }}$, where the spectrum of $\gamma$ shows a strong, spiked feature. It is argued that the observed discrepancy is of instrumental origin, due to the particularly strong dependence of $\gamma$ on the experimental uncertainties on $\sigma_{\text {tot }}$ whenever the latter is vanishingly small. Indeed, with a realistic uncertainty for $\sigma_{\text {tot }}$ near its minimum, the agreement between theory and experiment becomes very satisfactory.

\section{Theoretical method}

The differential photoionization cross-section of helium, with the parent ion $\mathrm{He}^{+}$being left in the state $\alpha$ and the photoelectron having linear momentum $\vec{k}_{e}$, is given by

$$
\frac{d \sigma_{\alpha}}{d \Omega}=\frac{4 \pi^{2}}{c \omega} \sum_{m_{\alpha} \sigma_{\alpha} \sigma}\left|\left\langle\psi_{\alpha, \vec{k}_{e} \sigma}^{(-)}\left|\hat{\epsilon} \cdot \sum_{i=1}^{2} e^{i \vec{k} \cdot \vec{r}_{i}} \vec{p}_{i}\right| \phi_{0}\right\rangle\right|^{2},
$$

where $\omega=c k$ is the photon energy (atomic units, $\hbar=1, m_{e}=1, e=1$, will be used throughout unless otherwise stated), $\hat{\epsilon}$ is the polarization of the impinging light, $\phi_{0}$ is the initial atomic state (average over the spin and spatial orientation of the initial state is omitted as we focus on the photoionization of the ${ }^{1} S$ ground state); $m_{\alpha}$ and $\sigma_{\alpha}$ are the magnetic quantum number and the spin projection of the residual electron in the parent ion $\alpha$; $\sigma$ is the spin projection of the photoelectron, and $\psi_{\alpha, \vec{k}_{e} \sigma}^{(-)}$is the final single-ionization scattering state, fulfilling incoming boundary conditions [8].

If the exponential in the transition operator in Eq. (1) is expanded in a Taylor series

$$
\exp (i \vec{k} \cdot \vec{r}) \simeq 1+i \vec{k} \cdot \vec{r}+\frac{1}{2}(i \vec{k} \cdot \vec{r})^{2}+\ldots
$$

the transition amplitude results in a sum of terms of increasing order in the photon wavenumber $k$ :

$$
\sum_{n=0}^{\infty} \frac{1}{n !}\left\langle\psi_{\alpha, \vec{k}_{e} \sigma}^{(-)}\left|\hat{\epsilon} \cdot \sum_{i=1}^{2}\left(i \vec{k} \cdot \vec{r}_{i}\right)^{n} \vec{p}_{i}\right| \phi_{0}\right\rangle .
$$

The ground state of helium is strongly localized, with a characteristic size of 1 a.u. Thus, for photon energies of the order of one atomic unit, the wavenumber $k$ is of the order of 0.01 a.u. and the series (3) converges rapidly. The dipole approximation is obtained by retaining the first term only (E1), which does not depend on $\vec{k}$, while the largest correction to the dipole approximation comes from the second term, linear in $k[9,10]$ which yield two terms identified as the magnetic dipole moment (M1) and the electric quadrupole moment (E2). The largest corrections to the photoionization cross section, beyond the pure electric dipole E1-E1, arise from the interference terms: electric dipole/magnetic dipole E1-M1 and electric dipole/electric quadrupole E1-E2. The correction to the photoelectron angular distribution due to these terms is proportional to $k$. If only the terms beyond the pure dipole which are 
linear in $k$ are retained, the partial differential cross section (PDCS) is fully described by just two additional nondipole parameters beyond the well known dipole total cross section $\sigma$ and asymmetry parameter $\beta$. Several equivalent parametrization of the PDCS have been proposed in the past $[2,10-17]$; the most common parametrization factors out the total cross section [15] and reads, for a linearly polarized radiation:

$$
\frac{d \sigma}{d \Omega}=\frac{\sigma_{\text {tot }}}{4 \pi}\left\{1+\beta \mathrm{P}_{2}(\cos \theta)+\left[\delta+\gamma \cos ^{2} \theta\right] \sin \theta \cos \phi\right\},
$$

where $\theta$ is the angle between the direction of observation $\hat{\Omega}$ and the polarization vector $\hat{\epsilon}$, while $\phi$ is the angle between the planes spanned by the vectors $(\hat{\epsilon}, \hat{k})$ and $(\hat{\epsilon}, \hat{\Omega})$. The case which is relevant here is the photoionization of the ground state of helium below the $N=2$ threshold. Since the ground state of $\mathrm{He}$ is an $\mathrm{S}$ state, $\beta=2$ identically and the transition matrix element due to the magnetic dipole is zero. The only contribution linear in $k$ comes from the E1-E2 interference term. According to the notation of the Appendix, the parameter $\gamma$ has the following expression

$$
\gamma=\frac{k \frac{-12 \sqrt{2} \pi^{2}}{c \omega} \operatorname{Re}\left(\left\langle\phi_{0}\left\|\hat{\mathbf{V}}_{1}^{0,0}\right\| \psi_{1 s E_{p}}^{(-) P_{0}^{o}}\right\rangle\left\langle\phi_{0}\left\|\hat{\mathbf{V}}_{2}^{1,0}\right\| \psi_{1 s E_{d}}^{(-)) D^{e}}\right\rangle^{*}\right)}{\sigma_{\text {tot }}}
$$

which is divergent at the possible zeros of $\sigma_{t o t}$. In the present case, if only the E1-E1 contribution to $\sigma_{\text {tot }}$ is retained, such divergence occurs close to each and every ${ }^{1} P^{o}$ resonance below the $\mathrm{N}=2$ threshold. It may be noted, however, that the measurement of the asymmetry parameter $\gamma$ involves two separate experimental determinations: that of the total cross section $\sigma_{t o t}$, on the one side, and that of the difference between the cross sections for the photoelectron being ejected in the forward and in the backward direction with respect to $\vec{k}$, on the other side. Therefore, to compare with experiments, the finite resolution of the experimental equipment should be taken into account. This was accomplished by the usual method of convoluting separately the calculated values of the numerator and of the denominator of eq. 5 with a normalized Gaussian function with an appropriate width. In fact, as detailed in the next section, a wide range of reasonable values for the experimental width were used. Yet, for energies very close to the minimum of $\sigma_{\text {tot }}$, the agreement between the resulting $\gamma(E)$ and the experiment did not show any sizeable improvement. Since, in the neighborhood of very small values of $\sigma_{t o t}, \gamma(E)$ is very sensitive to the accurate evaluation of the total cross section, the attention was focused on the possible shortcomings of the expression used to calculate $\sigma_{\text {tot }}$. As discussed in the next section, in order to improve the agreement with the experiment, the value of $\sigma_{\text {tot }}$ close to its minimum should be larger than its dipolar value. Thus, we considered those terms in $\sigma_{\text {tot }}$ which are neglected in the DA. The largest contributions arise from: i) the terms proportional to $k^{2}$; ii) the singlet component of the dominant ${ }^{3} \mathrm{P}_{J=1}^{o}$ triplet state due to spin-orbit coupling. With the notation introduced in the appendix, the expression for the total cross section truncated after the electric quadrupole contribution and retaining only the terms up to $k^{2}$ is

$$
\begin{aligned}
\sigma_{t o t} & =\frac{4 \pi^{2}}{3 c \omega}\left[\left|\left\langle\phi_{0}\left\|\hat{\mathbf{V}}_{1}^{0,0}\right\| \psi_{1 s E_{p}}^{(-) P^{o}}\right\rangle\right|^{2}+\frac{9}{10} k^{2}\left|\left\langle\phi_{0}\left\|\hat{\mathbf{V}}_{2}^{1,0}\right\| \psi_{1 s E_{d}}^{(-) D^{e}}\right\rangle\right|^{2}\right. \\
& \left.+2 k^{2} \Re\left\langle\phi_{0}\left\|\hat{\mathbf{V}}_{1}^{0,0}\right\| \psi_{1 s E_{p}}^{(-) P^{o}}\right\rangle\left\langle\psi_{1 s E_{p}}^{(-) P^{o}}\left\|\hat{\mathbf{V}}_{1}^{0,1}+\frac{1}{\sqrt{2}} \hat{\mathbf{V}}_{1}^{2,0}\right\| \phi_{0}\right\rangle\right] .
\end{aligned}
$$

In the energy region where the pure electric dipole amplitude $\mathrm{E} 1\left\langle\phi_{0}\left\|\hat{\mathbf{V}}_{1}^{0,0}\right\| \psi_{1 s E_{p}}^{(-) P^{o}}\right\rangle$ is vanishingly small, the second line in Eq. (6) can be neglected, so that the most important contribution is due to the E2-E2 term $\left|\left\langle\phi_{0}\left\|\hat{\mathbf{V}}_{2}^{1,0}\right\| \psi_{1 s E_{d}}^{(-) D^{e}}\right\rangle\right|^{2}$. The contribution of the higher multipoles to the total cross section can of course be safely ignored. As mentioned above, another possible contribution to the total cross section may come from the mixing between closely spaced doubly excited states of different multiplicity, due to the spin-orbit interaction [18-24]. It is well known, for example, that high values of $\ell$ for the external 
electron may induce a sizable mixing of the triplet and singlet manifolds $[25,26]$. In the present case, though, the orbital angular momentum involved is essentially $\ell=1$, so the spin-orbit mixing is rather small. If $c_{1}$ and $c_{2}$ are the coefficients of the ${ }^{1} \mathrm{P}^{o}$ and of the ${ }^{3} \mathrm{P}^{o}$ in the dominant ${ }^{3} \mathrm{P}_{J=1}^{o}$ state around an isolated resonance, $c_{1}$ is proportional to the ratio between the spin-orbit matrix element between resonances with different multiplicity and the energy difference between the closest singlet and triplet doubly excited states. The order of magnitude of the former is arguably the same as the fine structure of the lowest ${ }^{1} \mathrm{P}_{J}^{o}$ multiplet, that is $\sim 10^{-5}$ a.u. [27], while the energy difference between doubly excited states for principal quantum numbers of the outer electron $n \leq 8$ is of the order of $10^{-2}$ a.u. [20]. This means that the triplet contribution to the background cross section can be estimated to be six orders of magnitude smaller than the singlet-dipole background, and is therefore completely negligible.

\section{Results and discussion}

The continuum wave functions necessary to evaluate the electric dipole and electric quadrupole amplitudes in Eqs. (5) were computed with the B-spline K-matrix method. In the following we will summarize it briefly. Full details can be found in the literature $[28,29]$.

A stationary wave function $\psi_{\alpha E}^{\mathcal{P}}$ in the continuum is written as linear combination of partial-wave channel (PWC) functions $\phi_{\beta E}$ and of localized channel (LC) functions $\phi_{j}$. In each PWC, a well defined state of the parent ion is coupled to a single-electron wave function with assigned angular momentum, to give the required total angular momentum and spin. The radial part of the single-electron function is otherwise free. The LC is formed by antisymmetrized products of localized orbitals, and it serves the purpose of completing the description of the many-particle wave function at short radii. The hamiltonian is separately diagonalized in all the PWC's and in the LC. In this way, a complete set of independent stationary solutions to the secular problem at any given total energy $E$ can be sought in the form

$$
\psi_{\alpha E}^{\mathcal{P}}=\phi_{\alpha E}+\sum_{\gamma} \sum d \epsilon \phi_{\gamma \epsilon} \frac{\mathcal{P}}{E-\epsilon} K_{\gamma \epsilon, \alpha E}
$$

where $\mathrm{K}$ is the off-shell reaction matrix [8]. The index $\alpha$ runs over all the channels that are open at the energy E, while the index $\gamma$ runs over all the available channels (open and closed) including the LC. The requirement $\left\langle\phi_{\beta \epsilon}|E-H| \psi_{\alpha E}^{\mathcal{P}}\right\rangle=0 \forall \beta, \epsilon$, translates into a system of integral equations for the off-shell $\mathrm{K}$ matrix, which can be discretized and solved numerically.

The scattering solutions $\boldsymbol{\psi}_{E}^{ \pm}=\left(\psi_{1 E}^{ \pm}, \ldots, \psi_{n E}^{ \pm}\right)$fulfilling outgoing/incoming boundary conditions are obtained through the transformation

$$
\boldsymbol{\psi}_{E}^{ \pm}=\boldsymbol{\psi}_{E}^{\mathcal{P}}[\mathbf{1} \pm i \pi \mathbf{K}(E)]^{-1}, \quad\left\langle\boldsymbol{\psi}_{E}^{ \pm} \mid \boldsymbol{\psi}_{E^{\prime}}^{ \pm}\right\rangle=\delta\left(E-E^{\prime}\right) \mathbf{1} .
$$

For the phase conventions on the $\psi^{ \pm}$states, see the appendix. Resonance parameters are determined from the positions of the poles of the on-shell scattering matrix $S=$ $(1-i \pi K)(1+i \pi K)^{-1}$ in the lower half of the complex energy plane.

The radial component of all the single-particle wave functions are expanded on a B-spline set. B-splines of order 7 and a maximum orbital angular momentum $\ell_{\max }=8$ ensured a good accuracy. The radial space is partitioned in a localized sector and in a continuum/Rydberg sector. The former is defined on a subset of the spline space where the knots are optimized so as to reliably represent short-range correlations and bound states of the parent ion. The latter is capable of representing both the oscillating tail of the continuum states and the long range behavior of the Rydberg satellites up to a principal quantum number $n \sim 20$. The localized sector comprises $33 s, 32 p, 31 d, 30 f, 29 g, 28 h, 27 i, 27 j, 27 k$ orbitals, confined within a radius $R_{1} \sim 70$ a.u. The diffuse states are such that the continuum part is reliably represented up to a radius $R_{2} \sim 170$ a.u. while the Rydberg satellites stretch outwards up to few hundreds a.u. 
Since photoelectron anisotropy parameters are known to be rather delicate quantities, it is important to ensure that all the calculated quantities that influence them are satisfactorily converged. To this end, an extensive set of pertinent results was checked by comparison with the most accurate theoretical and experimental data available in the literature.

Bound states The energies of the hydrogen-like parent ions involved are virtually exact (within the electrostatic approximation), the error with respect to the hydrogen-like series for all the states involved being less than $2 \cdot 10^{-10}$ a.u. The energy of the ground state is $E=-2.903679895$ a.u. (accurate electrostatic limit -2.903724377 a.u. [30]). This value is intermediate between the accurate extrapolated limit for $\ell_{\max }=7, E=-2.90367459$ and for $\ell_{\max }=8, E=-2.90368947$ a.u. [31].

To ascertain the capability of the basis set to represent the far reaching tails of the more excited Rydberg states and, arguably, of the doubly excited states below the $\mathrm{N}=2$ threshold, the energies of the first ten Rydberg states in the ${ }^{1} \mathrm{~S},{ }^{1} \mathrm{P}^{o},{ }^{1} \mathrm{D}^{e}$ manifolds were compared with accurate electrostatic values reported in the literature [32] (par. 11.3). Since the values agree up to the ninth digit, the long range tail of the Rydberg satellites is certainly well represented.

Another stringent test is the comparison of the oscillator strengths for the transitions between the ground state and the first few ${ }^{1} \mathrm{P}^{o}$ Rydberg states with the corresponding most accurate values available in the literature [32] (par. 11.5). Also for these quantities, a very satisfactory agreement, to the fifth or sixth digit, is found. Furthermore, the matrix elements obtained in the length and acceleration gauges differ from those in the velocity gauge, on average, by only $\sim 0.003 \%$ and $\sim 0.01 \%$ respectively. Therefore, it can be concluded that, at least for the bound states, even the correlated short range part of the wave function is accurately represented.

Doubly excited states The resonance series are classified according to the Stark-Lin notation $\left[N_{1} N_{2} m\right]^{A}$. See the original paper by Lin [33] for tables of approximate quantum numbers for the autoionizing series in helium, and the review by Rost et al [34] for a comparison between different classification schemes. The resonances of the ${ }^{1} \mathrm{P}^{o}$ and of the ${ }^{1} \mathrm{D}^{e}$ manifolds below the $N=2$ threshold were calculated up to energies very close to the threshold $(n \sim 15)$. The energies and widths of the autoionizing states are obtained with a fit of the total phaseshift as a function of the energy, as detailed in [35]. As an example, in Tables 1 and 2 our results for the first few $[001]^{+}$and $[010]^{-}$resonances of the ${ }^{1} P^{o}$ manifold and the first few $[010]^{+}$and $[001]^{0}$ resonances of the ${ }^{1} D^{e}$ manifold are compared with the best data from the literature. A similar accuracy is found also for the higher terms $(n \leq 15)$ in all the ${ }^{1} \mathrm{P}^{o}$ and ${ }^{1} \mathrm{D}^{e}$ resonance series. The much narrower $[100]^{0}{ }^{1} \mathrm{P}^{o}$ and ${ }^{1} \mathrm{D}^{e}$ resonances are not listed since, at the experimental resolutions considered in this work, they are not visible. On the other hand, for photon energies above $64 \mathrm{eV}$, the resonant features in the nondipole anisotropy parameter are essentially a repetition of those between $62 \mathrm{eV}$ and $64 \mathrm{eV}$. Therefore, the listed resonances are sufficient to interpret all the qualitative features of the spectra examined in the present work. The agreement of energies, widths, and the q parameters of the Fano profiles is very good $\longrightarrow[. .$.$] (the energies, both from our calculations and from$ the literature, which were available in atomic units, have been converted to electronvolts by means of the transformation $\mathrm{E}(\mathrm{eV})=(\mathrm{E}(\mathrm{a} . \mathrm{u})+2.90369376) *$.27.20765438 , where 27.20765438 is the Rydberg constant for the $\mathrm{He}^{+}-\mathrm{e}^{-}$system).

Photoionization spectra As anticipated in the the previous section, the $\gamma$ parameter is particularly sensitive to the precise value of $\sigma_{t o t}$ in the neighborhood of the total cross section minima. For this reason, we also report the parameter $\sigma_{d r a g}$, defined as

$$
\sigma_{\text {drag }}=\hat{k} \cdot \int \frac{d \sigma}{d \Omega} \hat{\Omega} d \Omega
$$


Table 1. Parameters for the first few $[001]^{+}$and $[010]^{-1} \mathrm{P}^{o}$ resonances in helium, below $\mathrm{N}=2$ threshold (in brackets we report the dominant configuration of the lowest lying resonance). The present values (first rows) are compared with those by Rost et al [34] and to the experimental values by Schulz et al (1996) [36] and by Domke et al (1996) [37].

\begin{tabular}{lllll}
{$\left[N_{1} N_{2} m\right]_{n}^{A}$} & Ref. & $\mathrm{E}(\mathrm{eV})$ & $\Gamma(\mathrm{meV})$ & $q$ \\
\hline$[001]_{2}^{+}$ & & & & \\
$(\sim 2 s 2 p)$ & {$[34]$} & 60.144650 & 37.359 & -2.770 \\
& {$[36]$} & $60.1503(40)$ & $37.6(2)$ & $-2.73(4)$ \\
& {$[37]$} & 60.147 & 37 & -2.75 \\
{$[001]_{3}^{+}$} & & 63.655354 & 8.194 & -2.580 \\
& {$[34]$} & 63.655261 & 8.195 & -2.58 \\
& {$[36]$} & $63.6575(30)$ & $8.3(5)$ & $-2.53(4)$ \\
{$[010]_{3}^{-}$} & {$[37]$} & 63.658 & 10 & -2.5 \\
& & 62.757719 & 0.10460 & -4.252 \\
& {$[34]$} & 62.757719 & 0.1046 & -4.25 \\
& {$[36]$} & $62.7610(20)$ & $0.11(2)$ & $-4.1(4)$ \\
& {$[37]$} & 62.758 & $0.5(3)$ & -3.5
\end{tabular}

Table 2. Parameters of the first few $[010]^{+}$and $[001]^{0}{ }^{1} \mathrm{D}^{e}$ resonances below the $\mathrm{N}=2$ threshold (in brackets we report the dominant configuration of the lowest lying resonance). The present values (first rows) are compared with experimental and theoretical results by other authors: Chen (1999) [38], Lindroth (1994) [39], Ho and Bhatia (1991) [40], and DeHarak et al [41] (experimental results).

\begin{tabular}{lllll}
{$\left[N_{1} N_{2} m\right]_{n}^{A}$} & Ref. & \multicolumn{1}{c}{$\mathrm{E}(\mathrm{eV})$} & $\Gamma(\mathrm{meV})$ & \multicolumn{1}{c}{$q$} \\
\hline$\left.{ }^{010}\right]_{2}^{+}$ & & & & \\
$\left(\sim 2 p^{2}\right)$ & {$[38]$} & 59.9048 & 64.319 & -0.030 \\
& {$[39]$} & 59.9044 & 64.26 & \\
& {$[40]$} & 59.9044 & 64.267 & \\
& {$[41]$} & $59.9038(8)$ & $52(21)$ & \\
{$[010]_{3}^{+}$} & & 63.5156 & 15.129 & 0.249 \\
& {$[38]$} & 63.5157 & 15.17 & \\
& {$[39]$} & 63.5156 & 15.1 & \\
& {$[40]$} & 63.51553 & 15.1 & \\
{$[001]_{3}^{0}$} & {$[41]$} & $63.515(9)$ & $12(8)$ & \\
& & 63.8636 & 0.54530 & -2.102 \\
& {$[38]$} & 63.8636 & 0.5491 & \\
& {$[39]$} & 63.864 & 0.544 & \\
& {$[40]$} & 63.86353 & 0.547 & \\
& {$[41]$} & $63.855(8)$ & $8(8)$ &
\end{tabular}


which quantifies the net photoelectron flux in the forward direction. In the simple case studied here, $\beta=2, \delta=0$, so $\sigma_{d r a g}=(4 \pi / 15) \sigma_{t o t} \gamma$ is the only anisotropy parameter required for an alternative parametrization of the differential cross section where the total cross section is not factored out:

$$
\frac{d \sigma}{d \Omega}=\frac{3}{4 \pi} \sigma_{t o t} \cos ^{2} \theta+\frac{15}{4 \pi} \sigma_{d r a g} \cos ^{2} \theta \sin \theta \cos \phi,
$$

and therefore it does not have the same strong dependence on the experimental uncertainties of $\sigma_{\text {tot }}$ as $\gamma$.

Fig. 1 shows all the quantities involved in the evaluation of the nondipole anisotropy parameter measured by Krässig et al [6]: the dipole total cross section (Fig. 1.a), the quadrupole total cross section (Fig. 1.b), the drag cross section (Fig. 1.c), and the $\gamma$ parameter itself (Fig. 1.d). In Fig. 1 each quantity has been convoluted with several Gaussian weight functions in order to simulate the effect of an experimental slit function on the measured quantities. The following fwhm have been used: 20, 30, 40, 50, $60 \mathrm{meV}$, the smaller widths corresponding to the sharpest profiles. In Fig. 1.d, the ratio in the expression (5) is taken after the numerator and the denominator are independently convoluted with the slit function, with a given fwhm, assuming a zero background. The present spectra for $\gamma$ are compared with the experimental values by Krässig et al [6], obtained by digitalizing the plot in the original paper (the error introduced by the digitalization is significantly smaller than the experimental error bars). In the total cross section shown in Fig. 1.a, only the well isolated Fano profile of the $[001]_{2}^{+1} \mathrm{P}^{o}$ resonance is evident. The closely lying $[010]_{2}^{+1} \mathrm{D}^{e}$ resonance is visible in the much smaller electric-quadrupole contribution to the total cross section, shown in Fig. 1.b. The effect of both resonances is, on the contrary, apparent on the drag cross section shown in Fig. 1.c as well as in the $\gamma$ reported in fig. 1.d.

For a closer comparison with the experimental $\gamma$ spectrum (Fig. 1.d), our theoretical prediction was shifted at lower energies by $10 \mathrm{meV}$, well within the energy uncertainty of $20 \mathrm{meV}$ declared by the authors. In order to separately assess the accuracy of the energy calibration of the present theoretical spectra, we compared the total cross section with the experimental spectra by Schultz et al [36], with an absolute uncertainty in the energy of just $1.7 \mathrm{meV}$, in the region of the $n=20,21,22$ multiplets, where the experimental points could be accurately extracted from the original published plots. With an energy shift of just 0.5 $\mathrm{meV}$, well below the experimental uncertainty, our spectrum is in excellent agreement with the experimental one, see Fig. 2. Experimental data of comparable accuracy at lower energies are not readily available, but it is not unreasonable to assume a similar precision therein as well.

The comparison of the calculated $\gamma$ with the experimental values of Krässig et al [6] shows a fairly good agreement except at the energies close to the minimum of $\sigma_{\text {tot }}$. In the small region between $60.15 \mathrm{eV}$ and $60.3 \mathrm{eV}$, and particularly above $60.2 \mathrm{eV}$, none of the present convoluted theoretical profiles is really in good agreement with the experimental data. In particular, the theoretical values corresponding to resolution of $20 \mathrm{meV}$, as declared in [6], display an exaggeratedly spiked behavior. This is most likely due to a considerable difference between the theoretical and the experimental values of the total cross section in that critical region.

A comparison between Figs. 1.a and 1.b shows that, on average, the dipole cross section dominates over the quadrupole total cross section by as much as four orders of magnitude, as expected. As a consequence, it turns out that, with the present experimental resolution, the contribution of the E2-E2 term to $\sigma_{\text {tot }}$ close to the minima of the latter is completely negligible with respect to the contribution due to the finite energy resolution itself. In fact, we estimated that in order to be able to resolve the contribution of the E2-E2 term, a fwhm of $1 \mathrm{meV}$ or less would be required. In conclusion, the contribution of the quadrupole cross section to the total cross section near its minima is not really of any relevance whenever the energy resolution, as in the present case, is worse than few $\mathrm{meV}$. The cause of the observed discrepancy must be sought elsewhere. 


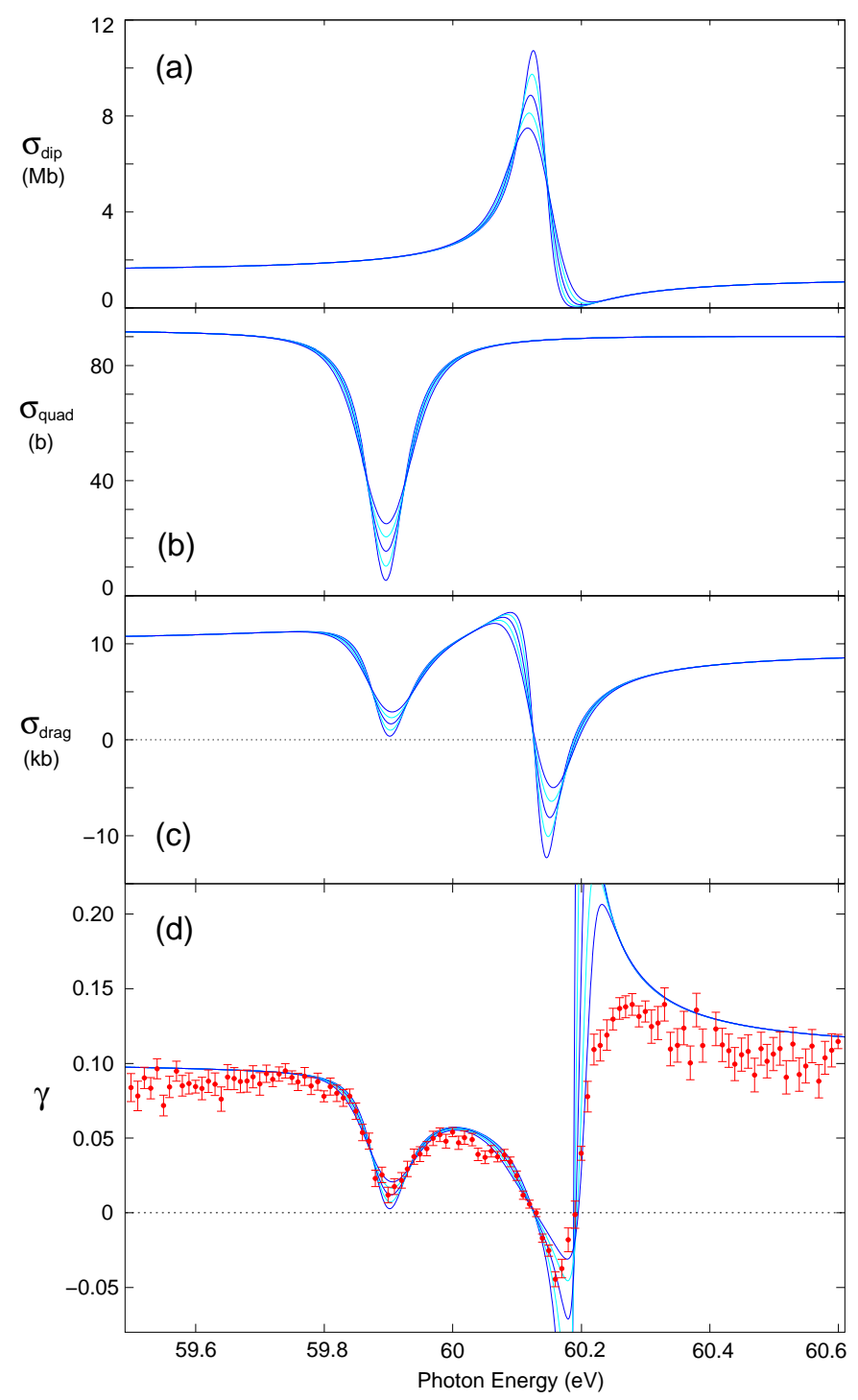

Figure 1. Parameters involved in the calculation of the nondipole asymmetry parameters in helium photoionization below the $\mathrm{N}=2$ threshold. a) dipole total cross section, b) quadrupole total cross section, c) drag cross section, d) nondipole asymmetry parameter $\gamma$. Each parameter has been convoluted with Gaussian weight functions with five different fwhm: 20, 30, 40, 50 and $60 \mathrm{meV}$. In d) the theoretical prediction is compared with the experimental data by Krassig et al [6], with a declared uncertainty of $20 \mathrm{meV}$. 


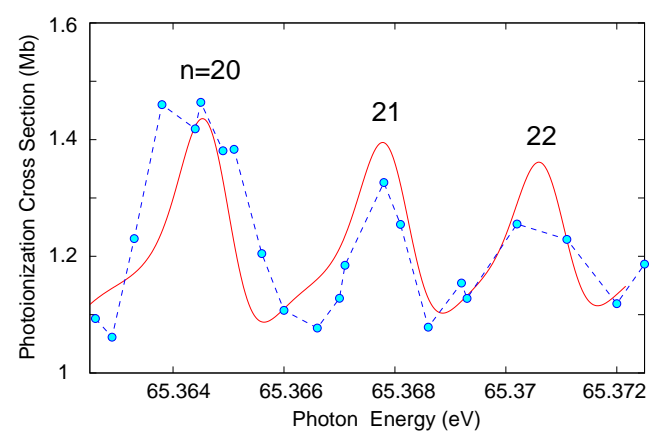

Figure 2. Energy calibration. Continuous (red) line: present results convoluted with a Gaussian function with $f w h m=1 \mathrm{meV}$ and shifted $0.5 \mathrm{meV}$ at higher energies. Dashed (blue) line/points: experimental data by Schulz et al digitalized from [36]. The agreement is very good as the uncertainty in the experimental energy calibration in this energy region is declared to be $1.7 \mathrm{meV}$.

A possible origin of the discrepancy may be a background signal of entirely instrumental origin. As a matter of fact, a background signal in the measurement of the total cross section due to experimental offsets, like stray light, is generally to be expected. For example, in a measurement of the helium total photoionization cross section below the $\mathrm{N}=2$ threshold performed in 2003 [42], a background cross section as large as $0.16 \mathrm{Mb}$ (i.e. just one order of magnitude smaller than the non-resonant cross section), is reported. In order to test the effect of a possible uncertainty in the experimental values of $\sigma_{\text {tot }}$, in Fig. 3 the experimental $\gamma$ parameter is compared with two theoretical curves, both with an energy resolution of $40 \mathrm{meV}$ : one which assumes no background (dashed curve, green on-line), the other which assumes a constant background equal to $0.2 \mathrm{Mb}$ (continuous curve, blue on-line), which is comparable to that reported in [42]. With the inclusion of such background, the large disagreement with the experimental results in the critical region disappears. Moreover, the inclusion of the background improves the agreement also in the non-resonant regions.

In [43] the measurement of $\gamma$ reported in [6] are compared to a semiempirical, convoluted profile for the non-dipole parameter. The resonant feature in the non-dipole amplitude used in such profile is obtained by including in the theoretical RPAE background a Fano profile whose parameters are taken from the literature. The shape of the bandpass is unspecified, while the resonant profile in the xenon spectrum reported in Fig.4 seems to confirm the existence of a non-zero background signal. In [43] the agreement found at photon energies around $60.2 \mathrm{eV}$ is good, while close to the the ${ }^{1} \mathrm{D}^{e}$ resonance large deviations are observed. At variance with this latter finding, our fully theoretical calculation is in very good agreement with the experiment in that region.

In the present case, near the energies where the total cross section almost vanishes, it may be presumed that the details of the peaks displayed by $\gamma$ might depend upon the experimental uncertainties of the energy resolution and of the background. The drag cross section reported in Fig. 1.c appears to be much less sensitive to the experimental resolution. In particular, it is not affected by the uncertainty on the offset of $\sigma_{t o t}$.

Sizable resonant effects in the nondipole asymmetry parameter due to the other terms belonging to the $[001]^{+}{ }^{1} \mathrm{P}^{o}$ and $[010]^{+}{ }^{1} \mathrm{D}^{e}$ series, as well as to the terms belonging to the $[010]^{-}{ }^{1} \mathrm{P}^{o}$ and $[001]^{0}{ }^{1} \mathrm{D}^{e}$, should be visible at higher energies. In Fig. 4, the dipole (a), the quadrupole (b), and the drag cross section (c) are reported below the $\mathrm{N}=2$ threshold, convoluted with a Gaussian weight function with $f w h m=5 \mathrm{meV}$. Already at this resolution, which is reasonable by today's standards, several other multiplets should be clearly visible. Figure 5 shows a detail of the same quantities close to the threshold. 


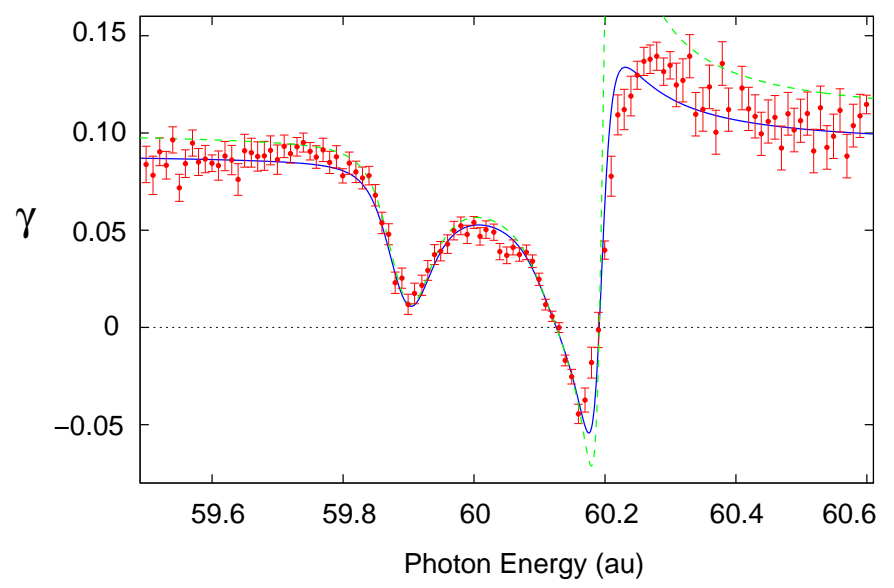

Figure 3. Nondipole anisotropy parameter $\gamma$ in helium in the neighborhood of the $[010]_{2}^{+}{ }^{1} \mathrm{D}^{e}$ electric quadrupole and of the $[001]_{2}^{+}{ }^{1} \mathrm{P}^{o}$ electric dipole resonance. The theoretical curves are evaluated with an energy resolution of 40 $\mathrm{meV}$ and assuming either a zero (dashed curve, green on-line) or a uniform $0.2 \mathrm{Mb}$ (continuous curve, blue on-line) background total cross section. The experimental data (points with error bars) are digitalized from Krässig et al [6].

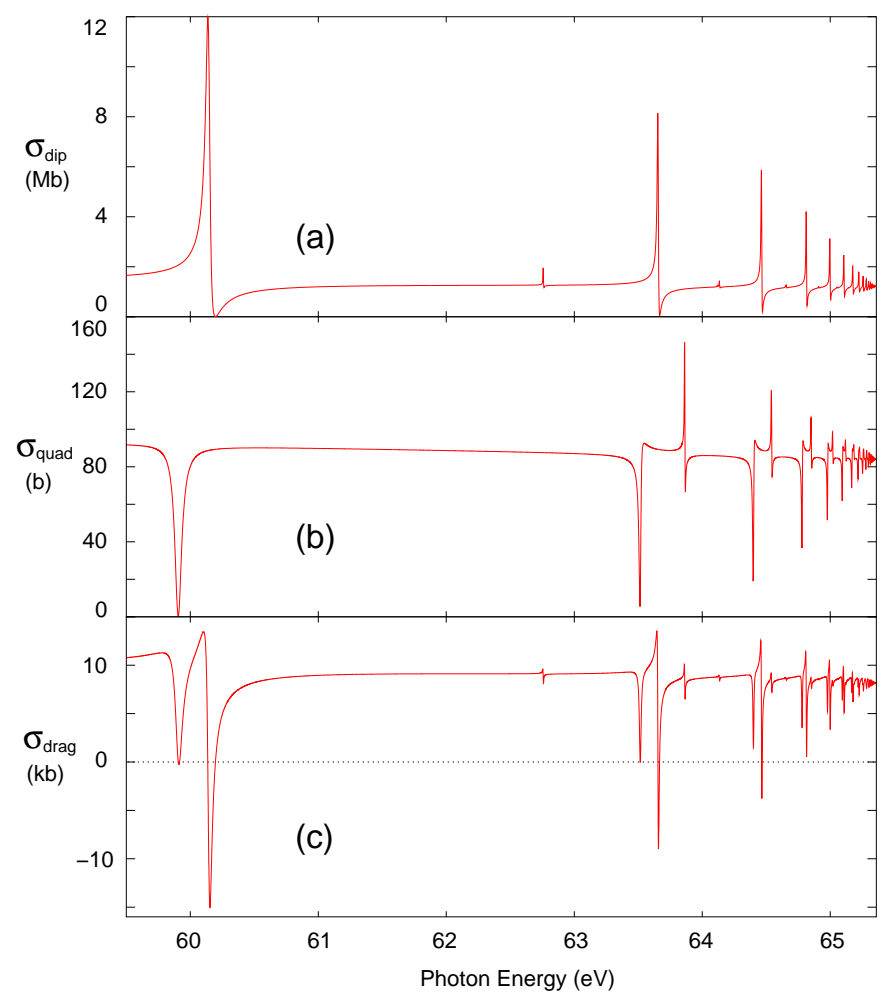

Figure 4. Dipole (a), quadrupole (b), and drag (c) cross sections below helium $\mathrm{N}=2$ threshold. 


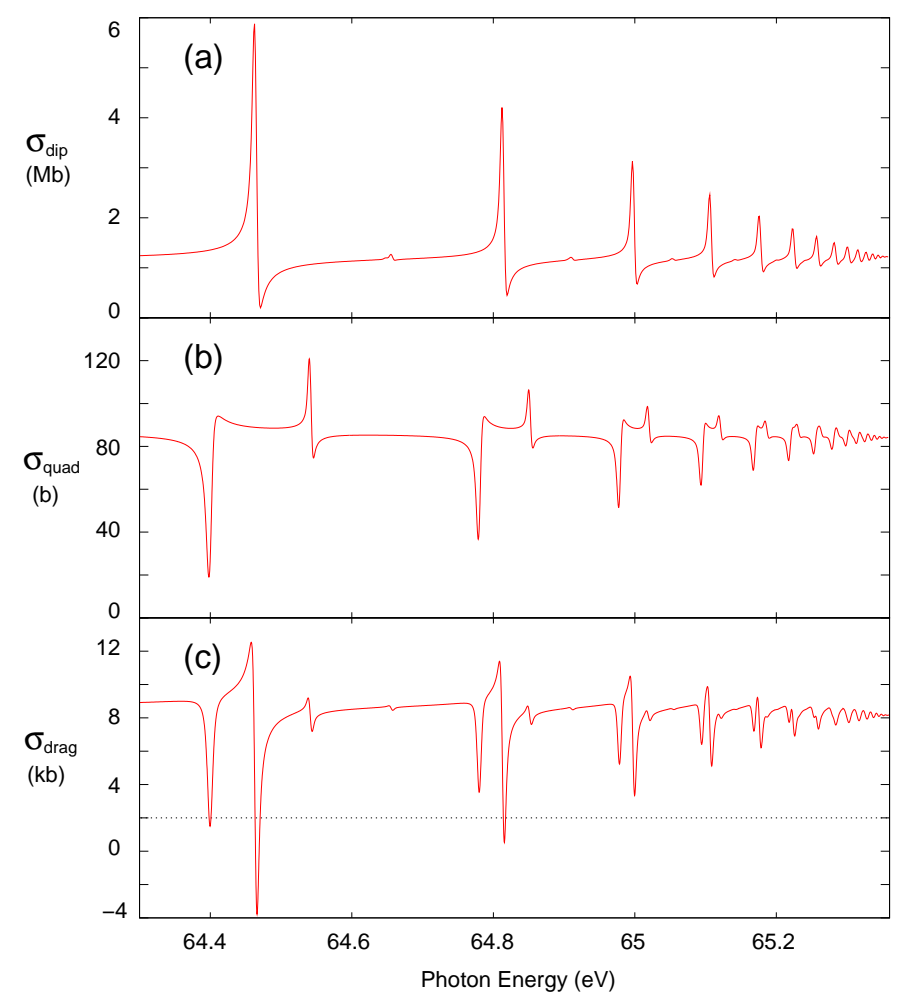

Figure 5. Detail of dipole (a), quadrupole (b), and drag (c) cross sections below helium $\mathrm{N}=2$ threshold.

\section{Summary}

We computed the nondipole anisotropy parameter $\gamma$ in the photoionization of helium below the $\mathrm{N}=2$ threshold; we compared our results with the experimental data by Krässig et al [6] between $59.5 \mathrm{eV}$ and $60.6 \mathrm{eV}$. In general, fairly good agreement between theoretical and experimental results was found, except near the minimum of the total photoionization cross section at $\sim 60.2 \mathrm{eV}$. An analysis of the approximations involved excluded any significant role of either electric quadrupole E2-E2 effects, or of the spin-orbit mixing between states of different multiplicity. The observed discrepancies might be instead attribuited to the experimental uncertainty in the total photoionization cross section around its minimum. In fact, because of its very definition, the nondipole parameter $\gamma$ is very sensitive to the uncertainties in the total cross section when the latter attains vanishingly small values. The agreement between theory and experiment is indeed completely restored when a realistic value for the background signal in the measurement of $\sigma_{\text {tot }}$ is assumed.

A similar behaviour of the nondipole anisotropy parameter is to be expected also at energies closer to the $\mathrm{N}=2$ threshold. In the present work, several resonant structures arising from the interplay between ${ }^{1} \mathrm{P}^{o}$ and ${ }^{1} \mathrm{D}^{e}$ doubly excited states have been calculated and should be experimentally detectable. For those cases which are similar to the one considered here, that is whenever the total cross section takes on values smaller than the instrumental sensitivity, it is advisable to use a parameter other than $\gamma$ (e.g. the drag photoionization cross section) to measure the forward/backward anisotropy of the photoelectron distribution. 


\section{Acknowledgments}

L.A. acknowledges the financial support by the Göran Gustafsson Foundation and the Swedish science research council (VR).

\section{Appendix A. Differential cross section}

In this appendix a general expression is derived for the atomic partial differential cross section for the absorption of one photon. The transition operator for the absorption of a photon by an atom, within the field of a monochromatic plane electromagnetic wave, is

$$
\mathcal{V}=\hat{\epsilon} \cdot \sum_{i=1}^{N} e^{i \vec{k} \cdot \vec{r}_{i}} \vec{p}_{i}
$$

where $\hat{\epsilon}$ indicates the polarization. Using the well known expansion of a plane wave in spherical components [44], Eq. (A.1) is readily expanded in electric $(J=T \pm 1)$ and magnetic $(J=T)$ multipoles:

$$
\mathcal{V}=\sum_{T=1}^{\infty} \sum_{\tau=-T}^{T} \sum_{J=T-1}^{T+1} \sqrt{4 \pi} \hat{\epsilon} \cdot \vec{Y}_{T}^{J \tau}(\hat{k}) \mathbf{V}_{T \tau}^{J},
$$

where the irreducible transition operator $\mathbf{V}_{T \tau}^{J}$ is defined as

$$
\mathbf{V}_{T \tau}^{J}=\sum_{i=1}^{N} \sqrt{4 \pi} i^{J} j_{J}\left(k r_{i}\right) Y_{T, \tau}^{J}(i) .
$$

Here $j_{J}(k r)$ is a spherical Bessel function [45], $Y_{J}^{M}=Y_{J M}^{*}$, and the vector spherical harmonics and tensor product conform to the following definitions $[44,46]$

$$
\begin{aligned}
& \vec{Y}_{T}^{J \tau}(\theta, \phi) \equiv \sum_{M \mu} C_{J M, 1 \mu}^{T \tau} Y_{J}^{M}(\theta, \phi) \hat{e}^{\mu}, \\
& Y_{T \tau}^{J}(i) \equiv \sum_{M m} C_{J M, 1 m}^{T \tau} Y_{J M}\left(\hat{r}_{i}\right) p_{1 m}^{(i)} .
\end{aligned}
$$

In the LS coupling when $\mathcal{V}$ is used in the expression for the partial differential cross section, Eq. (1), the partial differential single photoionization cross section, averaged over the orientations of the initial target state $\phi_{0}$ and summed over the orientations of the final parent ion state $\alpha$ and the spin projection of the photoelectron, may be written as

$$
\begin{gathered}
\frac{\partial \sigma_{\bar{\alpha}, E \hat{k}_{e}}}{\partial \Omega_{\hat{k}_{e}}}=\frac{(2 \pi)^{2}}{c \omega} \sum_{\ell_{e} m_{e}} Y_{\ell_{e} m_{e}}\left(\hat{k}_{e}\right) \sum_{T T^{\prime}} \sum_{J J^{\prime}}\left\{\sum_{\tau \tau^{\prime}} C_{\ell_{e} m_{e} T^{\prime} \tau^{\prime}}^{T \tau}\left[\vec{\epsilon} \cdot \vec{Y}_{T}^{J \tau}(\hat{k})\right]\left[\vec{\epsilon} \cdot \vec{Y}_{T^{\prime}}^{J^{\prime} \tau^{\prime}}(\hat{k})\right]^{*}\right\} \times \\
\times \sum_{\ell \ell^{\prime}} \sum_{L L^{\prime}} \frac{\sqrt{4 \pi} \Pi_{\ell \ell_{e} L L^{\prime}}}{\left(2 L_{0}+1\right) \Pi_{T}} C_{\ell 0 \ell_{e} 0}^{\ell^{\prime} 0}\left\{\begin{array}{ccc}
L^{\prime} & \ell_{e} & L \\
\ell & L_{\alpha} & \ell^{\prime}
\end{array}\right\}\left\{\begin{array}{ccc}
T & \ell_{e} & T^{\prime} \\
L^{\prime} & L_{0} & L
\end{array}\right\}(-)^{L+L^{\prime}+L_{0}+T^{\prime}+L_{\alpha}+\ell} \times \\
\times\left\langle\phi_{0}\left\|\mathbf{V}_{T}^{J}\right\| \psi_{\alpha E \ell}^{(-) \Gamma}\right\rangle \cdot\left\langle\phi_{0}\left\|\mathbf{V}_{T^{\prime}}^{J^{\prime}}\right\| \psi_{\alpha E \ell^{\prime}}^{(-) \Gamma^{\prime}}\right\rangle^{*},
\end{gathered}
$$

where $\psi_{\alpha E \ell}^{(-) \Gamma}$ is a suitably normalized continuum wave function with incoming boundary conditions, energy $E$, total angular momentum $L$, parity $\Pi$ and spin $S$ specified by the collective index $\Gamma$. To be explicit, if the parent ion $\alpha$, in state $\phi_{\alpha}$, has charge $Z$ and energy $E_{\alpha}, \psi_{\alpha E \ell}^{(-) \Gamma}$ is defined in such a way that, for large values of one of the $N$ electron radial variables, say $r_{N}$, its only outgoing component is

$$
\left[\psi_{\alpha E \ell}^{(-) \Gamma}\right]_{\text {outgoing }} \sim \sqrt{\frac{2 k}{\pi}} \Phi_{\alpha, \ell}^{\Gamma} \frac{e^{i k r_{N}+i \frac{Z}{k} \ln 2 k r_{N}}}{2 i k r_{N}} .
$$


Here $k=\left[2\left(E-E_{\alpha}\right)\right]^{1 / 2}$ and $\Phi_{\alpha, \ell}^{\Gamma}$ is obtained by coupling $\phi_{\alpha}$ to the angular and spin component of the $N$-th electron so as to give a total symmetry $\Gamma$.

In the total cross section, only the square moduli of the individual multipoles appear:

$$
\begin{gathered}
\sigma_{\alpha, E}=\frac{2 \pi^{2}}{c \omega\left(2 L_{0}+1\right)} \sum_{T L \ell_{\alpha}}\left\{\left|\sqrt{\frac{T}{2 T+1}}\left\langle\phi_{0}\left\|\mathbf{V}_{T}^{T+1}\right\| \psi_{\alpha E \ell_{\alpha}}^{(-) \Gamma}\right\rangle+\sqrt{\frac{T+1}{2 T+1}}\left\langle\phi_{0}\left\|\mathbf{V}_{T}^{T-1}\right\| \psi_{\alpha E \ell_{\alpha}}^{(-) \Gamma}\right\rangle\right|^{2}+\right. \\
\left.+\left|\left\langle\phi_{0}\left\|\mathbf{V}_{T}^{T}\right\| \psi_{\alpha E \ell_{\alpha}}^{(-) \Gamma}\right\rangle\right|^{2}\right\}
\end{gathered}
$$

where the first square modulus refers to the electric multipoles, while the second refers to the magnetic multipoles.

For photoionization processes in which only photons with large wavelengths are involved, the transition matrix elements can be conveniently expanded in powers of the argument $k r$ of the spherical Bessel function [45]

$$
\begin{aligned}
& \mathbf{V}_{T \tau}^{J}=\sum_{\nu=0}^{\infty} \mathbf{V}_{T \tau}^{J, \nu} k^{J+2 \nu} \quad \text { where } \\
& \mathbf{V}_{T \tau}^{J, \nu}=\sum_{i=1}^{N} \frac{\sqrt{4 \pi} i^{J} r_{i}^{J+2 \nu} Y_{T, \tau}^{J}(i)}{(-2)^{\nu} \nu !(2 J+2 \nu+1) ! !} .
\end{aligned}
$$

\section{References}

[1] Krause M O 1969 Phys. Rev. 177151

[2] Cooper J W and Manson S T 1969 Phys. Rev. 177157

[3] Wuilleumier F and Krause M O 1974 Phys. Rev. A 10242

[4] Dolmatov V K and Manson S T 1999 Phys. Rev. Lett. 83939

[5] Wang M S, Kim Y S, Pratt R H and Ron A 1982 Phys. Rev. A 25857

[6] Krässig B, Kanter E P, Southworth S H, Guillemin R, Hemmers O, Lindle D W, Wehlitz R and Martin N L S 2002 Phys. Rev. Lett. 88203002

[7] Hemmers O, Guillemin R and Lindle D W 2004 Rad. Phys. Chem. 70123

[8] Newton R G 2002 Scattering Theory of Waves and Particles (Dover Publications, Inc., Mineola, N.Y.)

[9] Bechler A and Pratt R H 1989 Phys. Rev. A 391774

[10] Bechler A and Pratt R H 1990 Phys. Rev. A 426400

[11] Amusia M Y, Arifov P U, Baltenkov A S, Grinberg A A and Shapiro S G 1974 Phys. Lett. A 4766

[12] Huang K N 1980 Phys. Rev. A 22223

[13] Huang K N 1982 Phys. Rev. A 263676

[14] Scofield J H 1989 Phys. Rev. A 403054

[15] Cooper J W 1990 Phys. Rev. A 426942

[16] Cooper J W 1992 Phys. Rev. A 453362

[17] Cooper J W 1993 Phys. Rev. A 471841

[18] Wills A A, Sokell E, Gorczyca T W, Feng X, Wiedenhoeft M, Canton S E and Berrah N 2002 J. Phys. B: At. Mol. Opt. Phys. 35 L367

[19] Lambourne J G et al. 2003 J. Phys. B: At. Mol. Opt. Phys. 364351

[20] Žitnik M, Bučar K, Štuhec M, Penent F, Hall R I and Lablanquie P 2002 Phys. Rev. A 65 032520

[21] Lambourne J G et al. 2003 J. Phys. B: At. Mol. Opt. Phys. 364339

[22] Martin N L S 2004 J. Phys. B: At. Mol. Opt. Phys. 374177

[23] Gorczyca T W, Rubensson J E, Såthe C, Ström M, Agåker M, Ding D, Stranges S, Richter R and Alagia M 2000 Phys. Rev. Lett. 851202

[24] Penent F, Lablanquie P, Hall R I, Žitnik M, Bučar K, Stranges S, Richter R, Alagia M, Hammond P and Lambourne J G 2001 Phys. Rev. Lett. 862758

[25] Hessels E A, Sturrus W G, Lundeen S R and Cok D R 1987 Phys. Rev. A 354489

[26] Snow E L, Komara R A, Gearba M A and Lundeen S R 2003 Phys. Rev. A 68022510

[27] Drake G W F and Martin W C 1998 Can. J. Phys. 76679

[28] Argenti L and Moccia R 2006 J. Phys. B: At. Mol. Opt. Phys. 392773

[29] Argenti L and Moccia R 2007 J. Phys. B: At. Mol. Opt. Phys. 403655 
[30] Freund D E, Huxtable B D and Morgan J D III 1984 Phys. Rev. A 29980

[31] Carroll D P, Silverstone H J and Metzger R M 1979 J. Chem. Phys. 714142

[32] Drake G W, ed. 1997 Atomic, Molecular, \& Optical Physics Handbook (Amer Inst of Physics, Leipzig)

[33] Lin C D 1984 Phys. Rev. A 291019

[34] Rost J M, Schulz K, Domke M and Kaindl G 1997 J. Phys. B: At. Mol. Opt. Phys. 304663

[35] Argenti L and Moccia R 2007 Th. Chem. Acc. 118485

[36] Schulz K, Kaindl G, Domke M, Bozek J D, Heimann P A, Schlachter A S and Rost J M 1996 Phys. Rev. Lett. 773086

[37] Domke M, Schulz K, Remmers G, Kaindl G and Wintgen D 1996 Phys. Rev. A 531424

[38] Chen M K 1999 Phys. Rev. A 602565

[39] Lindroth E 1994 Phys. Rev. A 494473

[40] Ho Y K and Bhatia A K 1991 Phys. Rev. A 442895

[41] deHarak B A, Childers J G and Martin N L S 2006 Phys. Rev. A 74032714

[42] Prince K C, Richter R, de Simone M, Alagia M and Coreno M 2003 Phys. Rev. A 68044701

[43] Kanter E P, Krässig B, Southworth S H, Guillemin R, Hemmers O, Lindle D W, Wehlitz R, Amusia M Y, Chernysheva L V and Martin N L S 2003 Phys. Rev. A 68012714

[44] Varshalovich D A, Moskalev A N and Khersonskii V K 1988 Quantum Theory of Angular Momentum (World Scientific, Singapore)

[45] Abramowitz M and Stegun I A 1964 Handbook of Mathematical Functions with Formulas, Graphs, and Mathematical Tables (Dover, New York) 9th edn (new york: dover); 10th edn (washington, dc: us govt printing office) edn.

[46] Manakov N L, Meremianin A V and Starace A F 2002 J. Phys. B: At. Mol. Opt. Phys. 3577 